\title{
ON EXTENSIONS OF TRIADS
}

\author{
YASUTOSHI NOMURA
}

Dedicated to the memory of Professor Tadasi Nakayama

\section{Introduction}

As an extension of a result due to W. D. Barcus and J. P. Meyer [4], T. Ganea [8] has recently proved a theorem concerning the fibre of the extension $E \cup C F \rightarrow B$ of a fibre map $p: E \rightarrow B$ to the cone $C F$ erected over the fibre $F$. In this paper we shall establish a generalized Ganea theorem which asserts that the homotopy type of the fibre of a canonical extension $\xi^{\prime}$ of a triad $A \stackrel{f}{\longrightarrow} Y \stackrel{g}{-} B$ (cf. [13]) is determined by those of $f$ and $g$ (see Theorem 3.4). This generalization yields a proof of a well-known theorem of Serre on relative fibre maps (see Corollary 3.9) and, as done by various authors (cf. [1], [10], [12]), a theorem of Blakers- Massey (see Corollary 4.4).

Our result can be used to derive a dual EHP sequence which generalizes a conditionally exact sequence established by G. W. Whitehead [15] and TsuchidaAndo [14]. The dual product introduced by M. Arkowitz ([2], [3]) allows us to describe the third homomorphism in that sequence.

Throughout this paper we will work in the category of spaces with basepoints, generally denoted by $*$, and based maps. Homotopies are assumed to respect base-points. The closed unit interval is denoted by $I$. Given a path $\omega: I \rightarrow X$ in $X$, we denote by $\omega_{u, v}$ the path defined by $\omega_{u, v}(t)=\omega((1-t) u+t v)$, where $0 \leqq \boldsymbol{u} \leqq v \leqq 1$. For paths $\omega, \tau$ with $\omega(1)=\tau(0)$, the path consisting of $\omega$ followed by $\tau$ will be denoted by $\omega+\tau$, and the inverse of $\omega$ by $-\omega$. As usual, $\Omega$ and $S$ are used, respectively, to denote the loop and suspension functors. $E X$ and $C X$ denote the space of paths in $X$ emanating from the base-point and the cone over $X$ respectively.

We are indebted to $T$. Ganea for sending us a preprint of [8].

Received June 21, 1965. 


\section{$\S 1$. Preliminaries}

Let $A \stackrel{f}{\longrightarrow} Y \stackrel{g}{\longleftarrow} B$ be a triad and let $E_{f, g}$ be its mapping track, as defined in [13], i.e.,

$$
E_{f, g}=\left\{(a, r, b) \in A \times Y^{1} \times B \mid f(a)=\gamma(0), g(b)=\gamma(1)\right\}
$$

with projections $P_{1}: E_{f, g} \rightarrow A, P_{2}: E_{f, g} \rightarrow B$. In particular, let $E_{f}^{-}$and $E_{g}$ be, respectively, the mapping track constructed for the triads $A \stackrel{f}{\longrightarrow} Y \stackrel{g}{\longleftarrow} *, * \longrightarrow Y$ $\stackrel{g}{\longleftarrow} B$, which are usually called the fibres of $f, g$.

Let $I: \Omega Y \rightarrow E_{f, g}$ be the natural injection. Then we have

LeMma 1.1. (see [13]) $I_{*}\left(\gamma_{1}\right)=I_{*}\left(\gamma_{2}\right)$ for $\gamma_{1}, \gamma_{2} \in \pi(V, \Omega Y)$ if and only if there exist $\alpha \in \pi(V, \Omega A), \beta \in \pi(V, \Omega B)$ such that $\gamma_{1}=(\Omega f)_{*}(\alpha)+\gamma_{2}+\left(\Omega_{g}\right)_{*}(\beta)$.

Now let $\gamma_{1}: E_{P_{2}} \rightarrow E_{f}, \gamma_{2}: E_{P_{1}} \rightarrow E_{g}$ be the maps induced by the following homotopy-commutative diagram

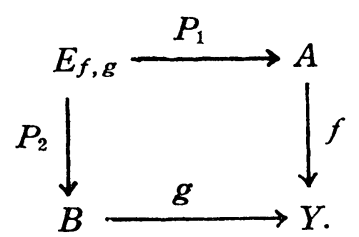

Lemma 1.2. (Dual excision theorem) $\chi_{1}$ and $\chi_{2}$ are homotopy equivalences.

Proof. We define $I_{2}: E_{g} \rightarrow E_{P_{1}}$ by $\Gamma_{2}(\beta, b)=(e ; *, \beta, b)$, where $e$ is the constant path at the base-point of $A$. Then it is easily seen that $\Gamma_{2}$ is a homotopy inverse of $\chi_{2}$.

Next let the diagram

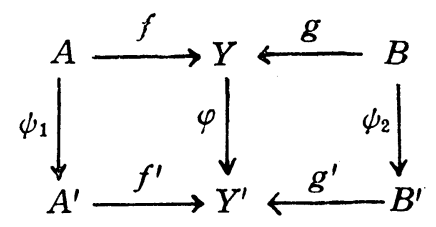

be homotopy-commutative. This induces the map $\chi: E_{f, g} \rightarrow E_{f^{\prime}, g^{\prime}}$ and the commutative diagram

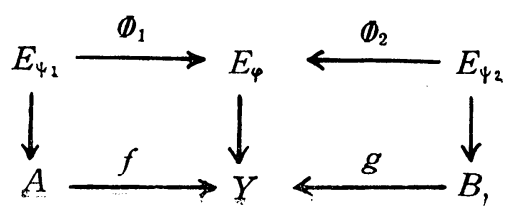


where the vertical maps are appropriate projections.

LEMMA 1.3. There exist a homeomorphism $\Xi: E_{\chi} \rightarrow E_{\Phi_{1}, \Phi_{2}}$ and an injection $l: \Omega E_{f^{\prime}, g^{\prime}} \rightarrow E_{\Phi_{1}, \Phi_{2}}$ such that the following diagram is homotopy commutative:

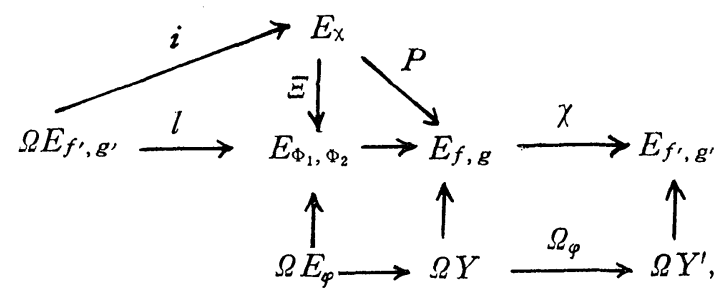

in which $i$ and $P$ are natural injection and projection, respectively. In particular, for a triple $A \stackrel{g}{\longrightarrow} B \stackrel{h}{\rightarrow} C$, the fibre of the natural map $\%: E_{h \circ g} \rightarrow E_{h}$ is of the same homotopy type as $E_{g}$.

Proof. It is sufficient to define $\Xi$ by setting

$$
\Xi(\alpha, \tilde{\gamma}, \beta ; a, \gamma, b)=((\alpha, a),(\tilde{\gamma} \circ \tilde{h}, \gamma),(\beta, b))
$$

for $a \in A, b \in B, \gamma \in Y^{l}, \alpha \in E A^{\prime}, \beta \in E B^{\prime}, \tilde{\gamma} \in E\left(Y^{\prime l}\right), \gamma(0)=f(a), \gamma(1)=g(b)$, $\alpha(1)=\phi_{1}(a), \beta(1)=\phi_{2}(b)$, where $\tilde{h}: I^{2} \rightarrow I^{2}$ is a homeomorphism indicated by the following picture:
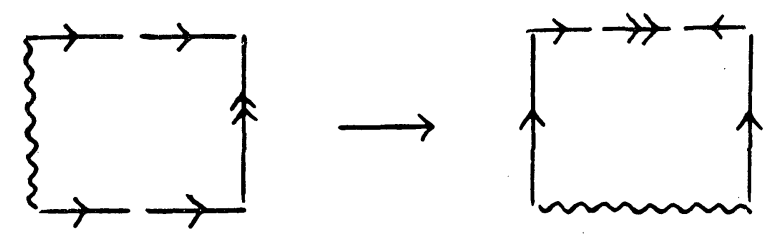

Now, let a cotriad $A \stackrel{f}{\stackrel{g}{\leftrightarrows}} X \stackrel{g}{\longrightarrow} B$ be given. We define its mapping cylinder $C_{f, g}$ to be the space obtained from $A \vee(X \times I) /(* \times I) \vee B$ by the identifications $f(x)=(x, 0), g(x)=(x, 1), x \in X$. The injections $I_{1}: A \rightarrow C_{f, g}, I_{2}: B \rightarrow C_{f, g}$ are obviously defined. The mapping cylinder of a cotriad $* \longleftarrow X \stackrel{g}{\longrightarrow} B$ is denoted by $C_{g}$, which is usually called the cofibre of $g$. Any point $x \in X$ defines a path $\hat{x}$ in $C_{f, g}, C_{g}$ or $S X$ which is given by

$$
\hat{x}(t)=(x, t), \quad 0 \leqq t \leqq 1 .
$$

Lemmas $1,1 \sim 1.3$ are dualized as follows: 
Lemma 1.1'. Let $Q: C_{f, g} \rightarrow S X$ be the map defined by shrinking $A$ and $B$ to a point. Then $Q^{*}\left(\gamma_{1}\right)=Q^{*}\left(\gamma_{2}\right)$ for $\gamma_{1}, \gamma_{2} \in \pi(S X, V)$ if and only if there exist $\alpha \in \pi(S A, V), \beta \in \pi(S B, V)$ such that $\gamma_{1}=(S f)^{*}(\alpha)+\gamma_{2}+(S g)^{*}(\beta)$.

Lemma 1.2'. (Excision theorem) Let $\chi_{1}^{\prime}: C_{f} \rightarrow C_{I_{2}}$ and $\chi_{2}^{\prime}: C_{g} \rightarrow C_{l_{1}}$ be the maps induced by the homotopy-commutative diagram

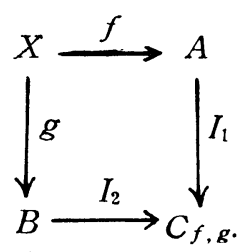

Then $\chi_{1}^{\prime}$ and $\chi_{2}^{\prime}$ are homotopy equivalences.

Lemma $1.3^{\prime}$. Let the diagram

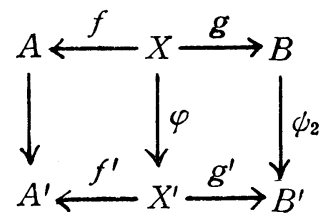

be homotopy-commutative, and let

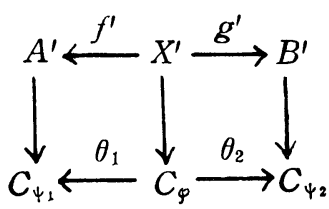

be the associated commutative squares. Then, for the mapping $\chi^{\prime}: C_{f, g} \rightarrow C_{f^{\prime}, g^{\prime}}$ induced by the above transformation, we have a homeomorphism $\Xi^{\prime}: C_{x^{\prime}} \rightarrow C_{\theta_{1}, \theta_{2}}$ such that the following diagram homotopy-commutes:

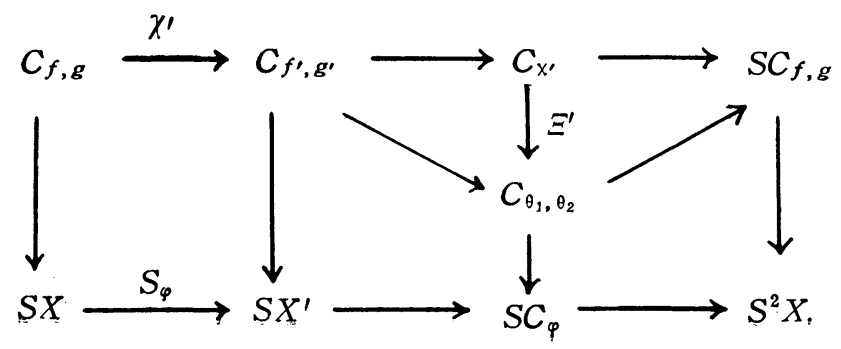


In particular, the cofibre of the natural map $C_{g} \rightarrow C_{h \circ g}$ induced by a triple $A \stackrel{g}{\longrightarrow} B \stackrel{h}{\longrightarrow} C$, is of the same homotopy type as $C_{h}$.

The following lemmas will be needed in the later sections.

Lemma 1.4. Let $\bar{f}: Y \rightarrow \Omega X$ be the map adjoint to $f: S Y \rightarrow X$, and suppose that $f$ and $X$ are, respectively, $m$ - and n-connected. Then $\bar{f}$ is $\min (2 n, m)$-connected.

Proof. By Lemma (4.1) of Berstein-Hilton [6], we have the commutative diagram

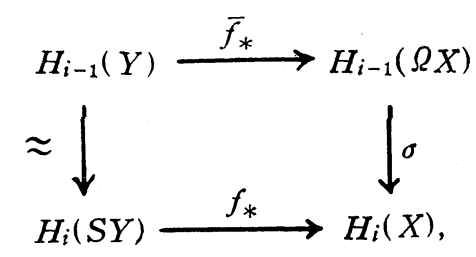

where $\sigma$ is the homology suspension. Since $\sigma$ is onto for $i \leqq 2 n+1$ and monomorphic for $i \leqq 2 n$, we obtain the desired conclusion.

Lemma 1.5. Suppose we are given $f: S Y \rightarrow X$ and its adjoint $\bar{f}: Y \rightarrow \Omega X$ and let $\vec{f}, Y$ be, respectively, $m$-, n-connected. Then $f$ is $[\min (m, 2 n+2)+1]$ connected.

Proof. It is sufficient to observe that, in the following commutative diagram, the homotopy suspension $E$ is onto for $i \leqq 2 n+2$ and monomorphic for $i \leqq 2 n+1$ :

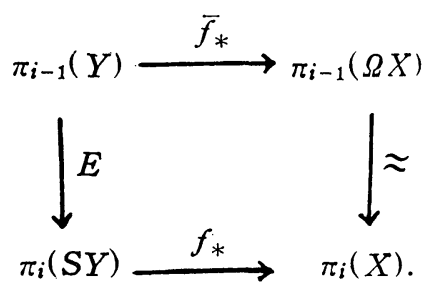

\section{$\S$ 2. Joins and cojoins}

Given a triad $A \stackrel{i_{1}}{\rightarrow} A \vee B \stackrel{i_{2}}{\leftarrow} B$ consisting of inclusions, we denote its mapping track $E_{i_{1}, i_{2}}$ by $A \hat{*} B$, which is called the cojoin of $A$ and $B$ (cf. [2]. Hilton uses the notation $A *^{\prime} B$ in $[9$, p. 238]). We have the diagram 


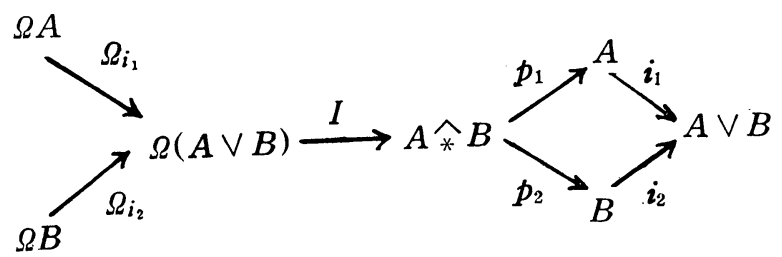

Let $A \mathrm{~b} B$ be the flat product of $A$ and $B$, i.e., the fibre $E_{J}$ of the injection $J: A \vee B \rightarrow A \times B$. Thus the sequence

$$
A \mathrm{~b} B \stackrel{L}{\rightarrow} A \vee B \stackrel{J}{\rightarrow} A \times B
$$

is essentially a fibre triple.

Lemma 2.1. $p_{1}$ and $p_{2}$ are null-homotopic.

Proof. Let $r: A \vee B \rightarrow A$ be the retraction resulting from shrinking $B$ to base-point. Note that $A \hat{*} B$ is the space of paths in $A \vee B$ which emanate from $A$ and end in $B$, and that $p_{1}$ is the fibre map which assigns to each path the starting point. Then we can readily see that a null-homotopy $p_{1} \simeq 0$ is generated by the correspondence $(\gamma, t) \rightarrow r \gamma(t), 0 \leqq t \leqq 1, \gamma \in A \hat{*} B$.

In the light of Lemma 2.1, we have exact rows in the following diagram

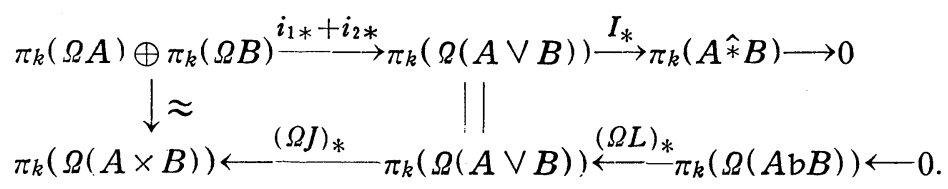

Since the composition $(\Omega J)_{*}{ }^{\circ}\left(i_{1 *}+i_{2 *}\right)$ is the direct sum representation, it follows by a routine argument (cf. [8, the proof of Theorem 3.2]) that $I_{* k} \circ(\Omega L)_{*}$ is bijective. Hence we have established

Proposition 2.2. ([2, p. 22]) $I \circ(\Omega L): \Omega(A \mathrm{bB}) \rightarrow A \hat{*} B$ is a weak homotopy equivalence.

Corollary 2.3. Suppose that $A$ is m-connected and $B$-connected. Then $A \hat{*} B$ is $(m+n-1)$-connected.

M. Arkowitz $([2,3])$ has defined the dual product $[\alpha, \beta]$ of $\alpha \in \pi(V, \Omega A)$ and $\beta \in \pi(V, \Omega B)$ to be the unique element $\gamma \in \pi(V, \Omega(A b B))$ such that $(\Omega L)_{*}$ $(\gamma)=-\left(\Omega i_{1}\right)_{*}(\alpha)-\left(\Omega i_{2}\right)_{*}(\beta)+\left(\Omega i_{1}\right)_{*}(\alpha)+\left(\Omega i_{2}\right)_{*}(\beta)$. Further, we denote the element $I_{*}\left(-\left(\Omega i_{2}\right)_{*}(\beta)+\left(\Omega i_{1}\right)_{*}(\alpha)\right) \in \pi(V, A \hat{*} B)$ by $\langle\alpha, \beta\rangle$, and call it the 
cojoin product of $\alpha$ and $\beta$. This is nothing but the second dual product $[\alpha, \beta]^{\prime}$ defined in [2].

Proposition 2.4. ([2, p. 22]) The weak homotopy equivalence $I \circ(\Omega L)$ sends $[\alpha, \beta]$ to $\langle\alpha, \beta\rangle$.

Proof. This is easily seen by noting, in view of Lemma 1.1, that

$$
\begin{aligned}
I_{*}\left(-\left(\Omega i_{1}\right)_{*}(\alpha)-\left(\Omega i_{2}\right)_{*}(\beta)+\left(\Omega i_{1}\right)_{*}(\alpha)\right. & \left.+\left(\Omega i_{2}\right)_{*}(\beta)\right) \\
& =I_{*}\left(-\left(\Omega i_{2}\right)_{*}(\beta)+\left(\Omega i_{1}\right)_{*}(\alpha)\right) .
\end{aligned}
$$

Now let $\bar{f}: V \rightarrow \Omega A, \bar{g}: V \rightarrow \Omega B$ be representatives of $\alpha, \beta$ and let $f: S V$ $\rightarrow A, g: S V \rightarrow B$ be adjoint to $\bar{f}, \bar{g}$ respectively. $f$ and $g$ obviously induce the map $f \hat{*} g: S V \hat{*} S V \rightarrow A \hat{*} B$. Let $\varepsilon: V \rightarrow \Omega S V$ be the natural injection defined by $\varepsilon(v)=\hat{v}, v \in V$, With these notations we have

Lemia 2.5. $\left(f^{*} g\right)_{*}\langle\varepsilon, \varepsilon\rangle=\langle\alpha, \beta\rangle$.

Proof. This follows from the fact that $\alpha=(\Omega f)_{*}(\varepsilon), \beta=(\Omega g)_{*}(\varepsilon)$ and from commutativity of the diagram

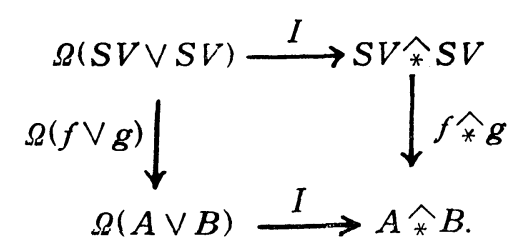

We mention here the relationship between the cup-product and the cojoin product. Let $A, B$ be the Eilenberg-MacLane spaces $K\left(G_{1}, p+1\right), K\left(G_{2}, q+1\right)$ respectively. Let

$$
\iota \in H^{p+q}(A \hat{*} B ; G) \approx \operatorname{Hom}\left(H_{p+q}(\Omega(A b B)) ; G\right) \approx \operatorname{Hom}(G, G)
$$

be the cohomology class corresponding to the identity homomorphism of $G$, where $G$ is the tensor product $G_{1} \otimes G_{2}$. Then Arkowitz [3] has proved

Proposition 2.6. $\langle\alpha, \beta\rangle^{*}(\iota)=\alpha \cup \beta$ for $\alpha \in H^{p}\left(V ; G_{1}\right), \beta \in H^{q}\left(V ; G_{2}\right)$.

Dually, the join $A * B$ of $A, B$ is defined to be the mapping cylinder $C_{p_{1}, p_{2}}$ of the cotriad $A \stackrel{p_{1}}{-} A \times B \stackrel{p_{2}}{\longrightarrow} B$, where $p_{1}, p_{2}$ are the projections. Any point of $A * B$ is represented by the symbol $(1-t) a \oplus t b, a \in A, b \in B, 0 \leqq t \leqq 1$. We have the diagram 


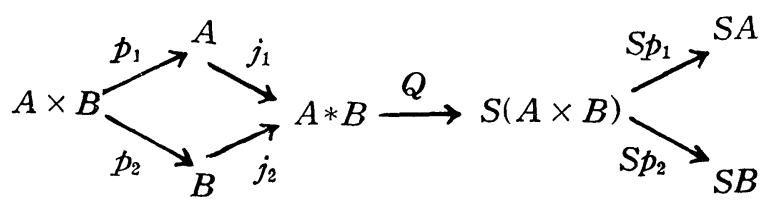

in which $j_{1} \simeq 0$ and $j_{2} \simeq 0$. Also, if we denote the cofibre of $A \vee B \stackrel{J}{\longrightarrow} A \times B$ by $A \sharp B$, we have a cofibre sequence

$$
A \vee B \stackrel{J}{\rightarrow} A \times B \stackrel{K}{\rightarrow} A \# B .
$$

Applying the same argument as in the proof of Proposition 2.2 to the diagram

$$
\begin{aligned}
& 0 \rightarrow H_{k}(A * B) \stackrel{Q_{*}}{\longrightarrow} H_{k}(S(A \times B)) \stackrel{\left\{\left(S p_{1}\right)_{*},\left(S p_{2}\right)_{*}\right\}}{\longrightarrow} H_{k}(S A) \oplus H_{k}(S B) \\
& 0 \leftarrow H_{\dot{k}}(S(A \# B)) \stackrel{(S K)_{*}}{\longleftarrow} H_{k}(S(A \times B)) \stackrel{(S J)_{*}}{\longleftarrow} H_{k}(S A \vee S B),
\end{aligned}
$$

we obtain.

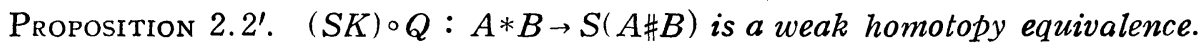

Now recall that the generalized Samelson product $\langle\langle\alpha, \beta\rangle\rangle \in \pi(S(A \vee B), V)$ of $\alpha \in \pi(S A, V)$ and $\beta \in \pi(S B, V)$ is defined to be the unique element $\gamma$ such that $q^{*}(\gamma)=-\left(S p_{1}\right)^{*}(\alpha)-\left(S p_{2}\right) *(\beta)+\left(S p_{1}\right) *(\alpha)+\left(S p_{2}\right) *(\beta)$ in the exact sequence

$$
0 \leftarrow \pi(S A \vee S B, V) \leftarrow \pi(S(A \times B), V) \stackrel{q^{*}}{-} \pi(S(A \wedge B), V) \leftarrow 0,
$$

where $A \wedge B$ is the smashed product $A \times B / A \vee B$ and $q: S(A \times B) \rightarrow S(A \wedge B)$ is the identification map. Note that, in this argument, $A$ and $B$ are assumed to have non-degenerate base-point. The generalized Whitehead product $[\alpha, \beta]$ is defined to be the element $Q^{*}\left(-\left(S p_{2}\right)^{*}(\beta)+\left(S p_{1}\right)^{*}(\alpha)\right) \in \pi(A * B, V)$. We see from Lemma $1.1^{\prime}$ that the homotopy equivalence $A * B \rightarrow S(A \wedge B)$ transforms $\langle\langle\alpha, \beta\rangle\rangle$ to $[\alpha, \beta]$.

We shall need, in $\S 5$, the map $W: \Omega A * \Omega B \rightarrow B \mathrm{~b} A$ which is defined by

$$
W((1-t) \alpha \oplus t \beta)= \begin{cases}\beta_{0,2} \times \alpha, & 0 \leqq 2 t \leqq 1, \\ \beta \times \alpha_{0,2-2}, & 1 \leqq 2 t \leqq 2 .\end{cases}
$$

for $\alpha \in \Omega A, \beta \in \Omega B$. Then the following lemma is well-known (cf. $[8, \S 2]$ ).

Lemma 2.8. W is a weak homotopy equivalence.

Dually, we define $W^{\prime}: A \# B \rightarrow S A \hat{*} S B$ as follows: 


$$
\begin{array}{r}
W^{\prime}(a, b)(t)= \begin{cases}(b, 1-2 t), & 0 \leqq 2 t \leqq 1, \\
(a, 2 t-1), & 1 \leqq 2 t \leqq 2,\end{cases} \\
W^{\prime}\left(a, b_{0}, s\right)(t)= \begin{cases}(a, 1-s), & 0 \leqq 2 t \leqq 1, \\
(a, 1-2 s+2 s t), & 1 \leqq 2 t \leqq 2,\end{cases} \\
W^{\prime}\left(a_{0}, b, s\right)(t)= \begin{cases}(b, 1-2 s t), & 0 \leqq 2 t \leqq 1, \\
(b, 1-s), & 1 \leqq 2 t \leqq 2,\end{cases}
\end{array}
$$

for $a \in A, b \in B, 0 \leqq s \leqq 1$. I regret to say that I was unable to show the dual of Lemma 2.8, but we will content ourselves with a partial result (see Corollary 5.10$)$.

\section{§ 3. Extensions of triads}

Let the diagram

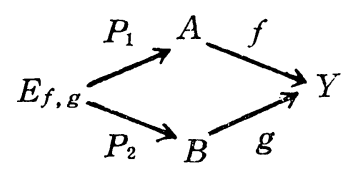

be associated with a triad $A \stackrel{f}{\longrightarrow} Y \stackrel{g}{\longleftarrow} B$, and consider the mapping cylinder $C_{P_{1}, P_{2}}$ of the cotriad $A \stackrel{P_{1}}{\longleftarrow} E_{f, g} \stackrel{P_{2}}{\longrightarrow} B$. We define the natural extension

$$
\xi^{\prime}: C_{P_{1}, P_{2}} \rightarrow Y
$$

of the triad $(f: g)$ over $C_{P_{1}, P_{2}}$ by setting

$$
\xi^{\prime}(a, \gamma, b ; t)=r(t), \xi^{\prime}(a)=f(a), \xi^{\prime}(b)=g(b)
$$

for $a \in A, b \in B, \gamma \in Y^{1}, 0 \leqq t \leqq 1$.

Next, let $f \nabla g: A \vee B \rightarrow Y$ be the map determined by $f$ and $g$, i.e., the composite $A \vee B \stackrel{f \vee g}{\longrightarrow} Y \vee Y \stackrel{\nabla}{\rightarrow} Y$, where $\nabla$ is the folding map. We define

$$
\eta^{\prime}: S E_{f, g} \rightarrow C_{f \nabla g}
$$

by setting, for $(a, \gamma, b) \in E_{f, g}, 0 \leqq s \leqq 1$,

$$
\eta^{\prime}(a, \gamma, b ; s)= \begin{cases}(a, 4 s) \in C A, & 0 \leqq 4 s \leqq 1, \\ \gamma\left(\frac{4 s-1}{2}\right) \in Y, & 1 \leqq 4 s \leqq 3, \\ (b, 4-4 s) \in C B, & 3 \leqq 4 s \leqq 4 .\end{cases}
$$

Introduce the homotopy-commutative diagram 


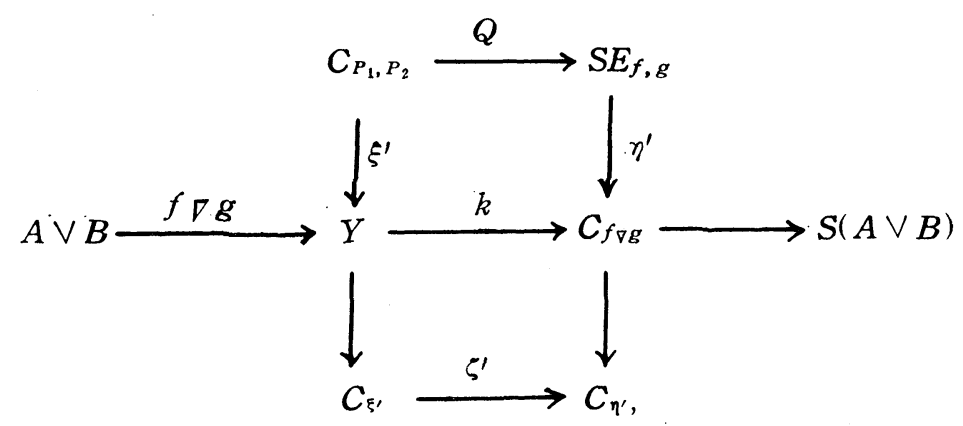

in which $\zeta^{\prime}$ is the map induced by the upper square and the unlabelled arrows denote the appropriate injections and identification.

The following proposition is an extension of Proposition 1.6 of Ganea [8].

Proposition 3.3. $\zeta^{\prime}: C_{\xi^{\prime}} \rightarrow C_{\eta^{\prime}}$ is a homotopy equivalence.

Proof. $\zeta^{\prime}$ is given explicitly as follows: if $2 s \leqq 1$, then

$$
\begin{aligned}
& \zeta^{\prime}(y)=y \in Y, \zeta^{\prime}(a, s)=*, \zeta^{\prime}(b, s)=*, \\
& \zeta^{\prime}(a, \gamma, b, t ; s)=(a, r, b, t ; 2 s) ;
\end{aligned}
$$

if $2 s \geqq 1$, then

$$
\begin{aligned}
& \zeta^{\prime}(y)=y \in Y, \zeta^{\prime}(a, s)=(a, 2 s-1) \in C_{f \nabla g}, \quad \zeta^{\prime}(b, s)=(b, 2 s-1), \\
& \zeta^{\prime}(a, \gamma, b, t ; s)= \begin{cases}(a, 4 t+2 s-1) \in C_{f \nabla g}, & 0 \leqq t \leqq \frac{1-s}{2} \\
r\left(\frac{2 t+s-1}{2 s}\right), & \frac{1-s}{2} \leqq t \leqq \frac{1+s}{2} \\
(b, 3+2 s-4 t) \in C_{f \nabla g}, & \frac{1+s}{2} \leqq t \leqq 1 .\end{cases}
\end{aligned}
$$

for cone parameter $s$ and cylinder one $t$. We consider $\varepsilon^{\prime}: C_{\eta^{\prime}} \rightarrow C_{\xi^{\prime}}$ given by

$$
\begin{aligned}
& \varepsilon^{\prime}(y)=y, \varepsilon^{\prime}(a, s)=(a, s), \varepsilon^{\prime}(b, s)=(b, s), \\
& \varepsilon^{\prime}(a, \gamma, b, u ; s)= \begin{cases}\left(a, r, b, \frac{1-s}{4} ; 4 u\right), & 0 \leqq 4 u \leqq s, \\
\left(a, \gamma, b, \frac{2 u(1+s)+1-2 s}{4-2 s} ; s\right), s \leqq 4 u \leqq 4-s, & 4-s \leqq 4 u \leqq 4\end{cases}
\end{aligned}
$$

for suspension parameter $u$. It is a troublesome but routine matter to verify that $\varepsilon^{\prime}$ is a homotopy inverse of $\zeta^{\prime}$. 
One of the main objects in this section is to prove the following theorem which generalizes Theorem 1.1 in [8].

THEOREM 3.4. The fibre $E_{\xi^{\prime}}$ of $\xi^{\prime}: C_{P_{1}, P_{2}} \rightarrow Y$ has the same homotopy type as the join $E_{f} * E_{g}$ of the fibres of $f$ and $g$.

Proof. We define $F: E_{\bar{f}} * E_{g} \rightarrow E_{\xi}$, and $G: E_{\xi} \rightarrow E_{f} * E_{g}$ by setting, for $a \in A, b \in B, \alpha, \beta, \gamma, \tau \in Y^{I}, 0 \leqq t \leqq 1$,

$$
\begin{aligned}
& \left\{\begin{array}{l}
F(a, \alpha)=(-\alpha ; a), F(\beta ; b)=(\beta ; b) \\
F((1-t)(a, \alpha) \oplus t(\beta, b))= \begin{cases}\left(-\alpha_{2} t, 1 ; a, \alpha+\beta, b, t\right), & 0 \leqq 2 t \leqq 1 \\
\left(\beta_{0,2 t-1} ; a, \alpha+\beta, b, t\right), & 1 \leqq 2 t \leqq 2,\end{cases}
\end{array}\right. \\
& G(\tau ; a)=\left(a, e_{\tau(1)}-\tau\right), G(\tau ; b)=\left(\tau+e_{\tau(1)}, b\right), \\
& G(\tau ; a, \gamma, b, t)=(1-t)\left(a, \gamma_{0, t}-\tau\right) \oplus t(\tau+\gamma t, 1, b),
\end{aligned}
$$

where $e_{x}$ denotes the constant path at $x$.

$G \circ F$ can be deformed into the identity via a homotopy $\Phi_{u}, 0 \leqq u \leqq 2$, whose value $\Phi_{u}((1-t)(a, \alpha) \oplus t(\beta, b))$ is given by setting, if $0 \leqq 2 t \leqq 1,0 \leqq u \leqq 1$,

$$
(1-t)\left(a, \alpha_{0,2}+\alpha_{2} t, 1\right) \oplus t\left(-\alpha_{2(1-u) t+u, 1}+(\alpha+\beta)_{(1-u) t+\frac{u}{2}, 1}, b\right) ;
$$

if $1 \leqq 2 t \leqq 2,0 \leqq u \leqq 1$,

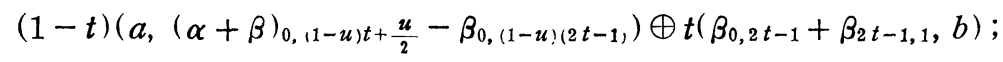

if $0 \leqq 2 t \leqq 1,1 \leqq u \leqq 2$,

$$
(1-t)\left(a, \alpha_{0,2 t(2-u)+\frac{u-1}{2}+\alpha_{2} t(2-u)+\frac{u-1}{2}, 1}\right) \oplus t\left(\beta_{0, \frac{u-1}{2}}+\beta \frac{u-1}{2}, 1, b\right) ;
$$

if $1 \leqq 2 t \leqq 2,1 \leqq u \leqq 2$,

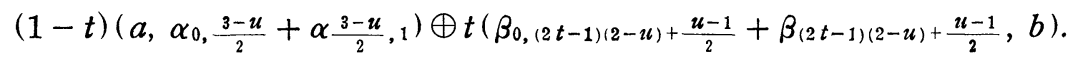

$F \circ G \simeq 1$ is verified by taking a homotopy $\Psi_{u}, 0 \leqq u \leqq 2$, whose value $\Psi_{u}(\tau ;$ $a, \gamma, b, t)$ is, if $0 \leqq u \leqq 1$,

$$
\left(\delta ; a,\left(\gamma_{0, t}-\tau\right)_{0,1-\frac{u}{2}}+\left(\tau+\gamma_{t, 1}\right) \frac{u}{2}, 1, b, t\right),
$$

where

$$
\delta= \begin{cases}-\left(\gamma_{0, t}-\tau\right)_{2 t-u t, 1} & 0 \leqq 2 t \leqq 1 \\ (\tau+\gamma t, 1)_{0,(1-t) u+2 t-1} & 1 \leqq 2 t \leqq 2\end{cases}
$$

if $1 \leqq u \leqq 2$, 


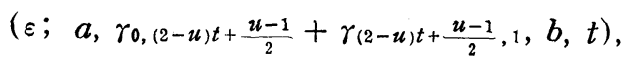

where

$$
\varepsilon=\left\{\begin{array}{cc}
-\left(\gamma_{0, t}-\tau\right)_{(2-u) t+\frac{u-1}{2}, 1} & 0 \leqq 2 t \leqq 1, \\
(\tau+\gamma t, 1)_{0,(2-u) t+\frac{u-1}{2}} & 1 \leqq 2 t \leqq 2 .
\end{array}\right.
$$

Thus the proof of Theorem 3.4 is complete.

The composition $E_{\bar{f}} * E_{g} \stackrel{F}{\longrightarrow} E_{\xi}, \longrightarrow C_{P_{1}, P_{2}}$ will be denoted by $j: E_{f} * E_{g} \rightarrow C_{P_{1}}, P_{2}$. This is given by

$$
j((1-t)(a, \alpha) \oplus t(\beta, b))=(a, \alpha+\beta, b ; t) .
$$

Consequently, the sequence

$$
E_{f}^{-} * E_{g} \stackrel{j}{\longrightarrow} C_{P_{1}, P_{2}} \stackrel{\xi^{\prime}}{\rightarrow} Y
$$

is essentially the fibre triple.

Combining Theorem 3.4 with Proposition 3.3 we obtain

COROLlary 3.7. Suppose that $f$ is p-connected and $g$ q-connected. Then $\xi^{\prime}$ and $\eta^{\prime}$ are both $(p+q+1)$-connected.

Remark As in Proposition 1.5 of [8], there exists a map $\Gamma: \Omega Y \rightarrow \Omega C_{P_{1}, P_{2}}$ such that $\Omega 5^{\prime} \circ \Gamma=$ identity. It is sufficient to define $I$ by $\Gamma(\omega)(t)=(*, \omega, * ; t)$. Note that the diagram

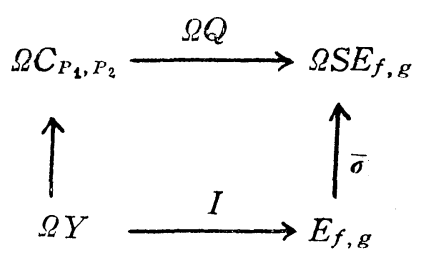

is commutative, in which $\vec{\sigma}$ is the canonical injection

Now we shall deduce the well known theorem of Serre on relative fibre maps from Corollary 3.7. For this purpose we prove.

THEOREM 3.8. Let $\Phi_{1}: C_{P_{1}} \rightarrow C_{g}$ and $\Phi_{2}: C_{P_{2}} \rightarrow C_{f}$ be the maps induced by the homotopy-commutative diagram (3.1). Then the cofibres of $\Phi_{1}$ and $\Phi_{2}$ have the same homotopy type as those of $\xi^{\prime}$.

Proof. Let the diagram 


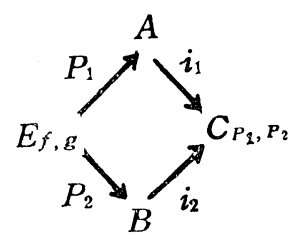

be associated with the cotriad $P_{1}, P_{2}$. Using this, the maps $\chi_{1}^{\prime}: C_{P_{1}} \rightarrow C_{i_{2}}$ and $\chi_{2}^{\prime}: C_{P_{2}} \rightarrow C_{i_{1}}$ are obviously defined. On the other hand, $\xi^{\prime}: C_{P_{1}, P_{2}} \rightarrow Y$ determines the maps $k_{1}: C_{i_{2}} \rightarrow C_{g}$ and $k_{2}: C_{i_{1}} \rightarrow C_{f}$. We see easily that the compositions $k_{1} \circ \chi_{1}^{\prime}$ and $k_{2} \circ \chi_{2}^{\prime}$ coincide with $\Phi_{1}$ and $\Phi_{2}$, respectively. Since both $C_{k_{1}}$ and $C_{k_{2}}$ are equivalent to $C_{\xi^{\prime}}$ by Lemma $1.3^{\prime}$, and since $\chi_{1}^{\prime}$ and $\chi_{2}^{\prime}$ are homotopy equivalences by Lemma $1.2^{\prime}$, we conclude that $C_{\Phi_{1}}$ and $C_{\Phi_{2}}$ are equivalent to $C_{\xi}$, which completes the proof.

Corollary 3.9. (Serre theorem on relative fibre maps) Suppose that $f$ is p-connected and $g$ q-connected, and that $g$ is a fibration. Let $\bar{\Phi}_{1}: C_{\pi_{1}} \rightarrow C_{g}, \bar{\Phi}_{2}$ : $C_{\pi_{2}} \rightarrow C_{f}$ be the maps determined by the commutative square:

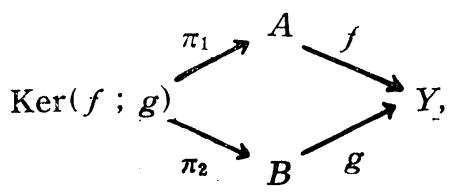

where $\operatorname{Ker}(f: g)$ is the fibre space induced from $g$ by $f$. Then $\bar{\Phi}_{1}$ and $\bar{\Phi}_{2}$ are $(p+q+1)$-connected.

This follows from Corollary 3.7 and Theorem 3.8, observing that $\bar{\emptyset}_{1}$ and $\bar{\emptyset}_{2}$ are, respectively, equivalent to $\Phi_{1}$ and $\Phi_{2}$ of Theorem 3.8 .

Theorem 3.10. Suppose that $f$ is p-connected and $g$ q-connected. Let $V$ be a 1-connected space such that $\pi_{i}(V)=0$ for $i \geqq p+q+1$. If $A, B, Y$ and $V$ have the homotopy type of $C W$-complexes, then the following sequence is exact:

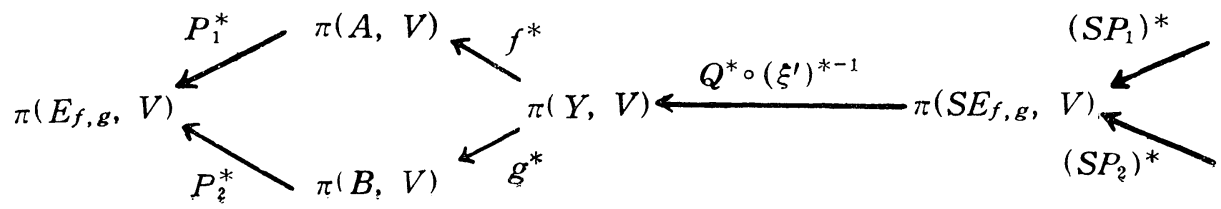




\section{§. Lifting cotriads}

Let $A \stackrel{f}{\longleftarrow} X \stackrel{g}{\longrightarrow} B$ be a cotriad and let

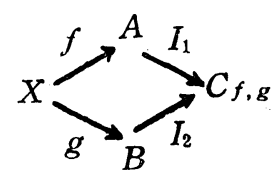

be the associated diagram. Consider the mapping track $E_{1_{1}, L_{2}}$ of the triad $I_{1}$, $I_{2}$ and let $f \Delta g: X \rightarrow A \times B$ be the composition $X \stackrel{\Delta}{\longrightarrow} X \times X \stackrel{f \times g}{\rightarrow} A \times B$, where $\Delta$ is the diagonal injection. We define $\xi: X \rightarrow E_{1_{1}, l_{2}}$ and $\eta: E_{f \Delta g} \rightarrow \Omega C_{f, g}$ by setting, for $x \in X, \alpha \in E A, \beta \in E B$,

$$
\begin{aligned}
& \xi(x)=(f(x), \hat{x}, g(x)), \quad \hat{x}(s)=(x, s) \in X \times I \subset C_{f, g} \\
& \eta(x, \alpha \times \beta)(s)= \begin{cases}\alpha(4 s), & 0 \leqq 4 s \leqq 1, \\
\left(x, \frac{4 s-1}{2}\right), & 1 \leqq 4 s \leqq 3, \\
\beta(4-4 s), & 3 \leqq 4 s \leqq 4 .\end{cases}
\end{aligned}
$$

Introduce the following homotopy-commutative diagram

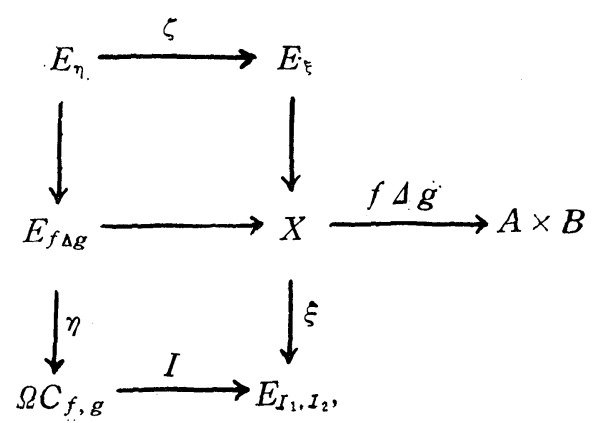

in which $I$ is the injection and $\zeta$ is the map induced by the lower (homotopycommutative) square

Proposition 4.2. $\zeta: E_{\eta} \rightarrow E_{\xi}$ is a homotopy equivalence.

As shown in [12], we can deduce the Blakers-Massey theorem on excisive triads from the Serre theorem on relative fibre maps. For this purpose we prove

TheOREM 4.3. Suppose that $f$ is p-connected and $g$ q-connected: Then $\xi$ and $\eta$ are $(p+q-1)$-connected.

Proof. We consider the homotopy-commutative diagram 


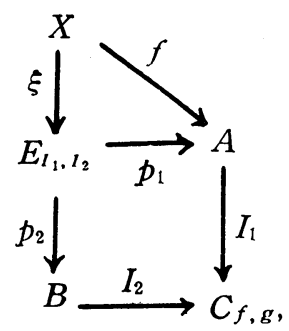

in which the square is associated with the triad $I_{1}, I_{2}$. By Lemma $1.2^{\prime}, I_{2}$ and $I_{1}$ are, respectively, $p$ - and $q$-connected. Applying Theorem 3.8 to the above square, the map

$$
\chi: C_{p_{1}} \rightarrow C_{l_{2}}
$$

induced by the above homotopy-commutative square, is $(p+q+1)$-connected.

Now it is easily seen that the composition

$$
C_{f} \longrightarrow C_{p_{1}} \stackrel{\chi}{\rightarrow} C_{I_{2}},
$$

in which the first map is determined by $\xi$, coincides with the homotopy equivalence $\chi_{1}^{\prime}: C_{f} \rightarrow C_{I_{2}}$ of Lemma 1.2'. Thus, $C_{f} \rightarrow C_{p_{1}}$ is $(p+q)$-connected, and therefore, by resorting to Proposition 4.2 and the sequence

$$
C_{\xi} \rightarrow C_{f} \rightarrow C_{p_{1}} \rightarrow S C_{\xi} \rightarrow S C_{f} \rightarrow S C_{p_{1}} \rightarrow \cdots,
$$

we can infer that $\xi$ and $\eta$ are $(p+q-1)$-connected.

Suppose further that $g$ is a cofibration and

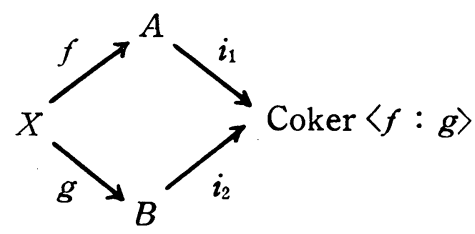

is the associated commutative diagram, where Coker $\langle f: g\rangle$ is the space obtained from $A \vee B$ by the identification $f(x)=g(x), x \in X$. Let

$$
\bar{\eta}: E_{f \Delta g} \rightarrow \Omega \text { Coker }\langle f: g\rangle
$$

be the map given by $\bar{\eta}=\Omega q \circ \eta$, where $q: C_{f, g} \rightarrow$ Coker $\langle f: g\rangle$ is the canonical equivalence. Note that, since $g$ is an inclusion, $E_{f \Delta g}$ can be identified with the space consisting of $(\alpha, \beta) \in E A \times E B$ such that $i_{1} \alpha(1)=i_{2} \beta(1)$, i.e., the space $S_{i_{1}, i_{2}}$ as defined in [13]. 
Since $\bar{\eta}$ is homotopic to $m: S_{i_{1}, i_{2}} \rightarrow \Omega$ Coker $\langle f: g\rangle$ which is given by

$$
m(\alpha, \beta)=\left(\Omega i_{1}\right)(\alpha)-\left(\Omega i_{2}\right)(\beta),
$$

and since the sequence

$$
\pi_{k}\left(\Omega^{2} \text { Coker }\langle f: g\rangle\right) \rightarrow \pi_{k}\left(T_{i_{1}, i_{2}}\right) \rightarrow \pi_{k}\left(S_{i_{1}, i_{2}}\right) \stackrel{m_{*}}{\rightarrow} \pi_{k}(\Omega \text { Coker }\langle f: g\rangle)
$$

is exact by Proposition 3.3 of [13], where $T_{i_{1}, i_{2}}$ is the subspace of $E A \times E B \times$ $E E$ Coker $\langle f: g\rangle$ consisting of $(\alpha, \beta, \tilde{\gamma})$ such that $\tilde{\gamma}(s, 1)=i_{1} \alpha(s), \widetilde{\gamma}(1, t)=i_{2} \beta(t)$, it follows

Corollary 4.4. (Blakers-Massey) If $f$ and $g$ are, respectively, $p$ - and $q$ connected, and if $g$ is a cofibration, then $T_{i_{1}, i_{2}}$ is $(p+q-2)$-connected.

Corollary 4.5. Suppose that $f$ is p-connected and $g$-connected. Then, for any $C W$ complex $V$ with $\operatorname{dim} V \leqq p+q-2$, the following sequence is exact:

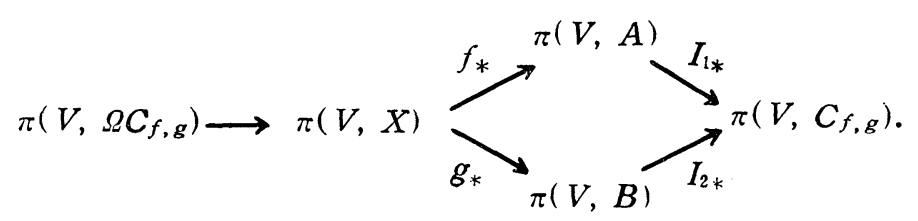

The dual of Theorem 3.8 is stated as follows:

THEOREM 4.6. Let $\Phi_{1}^{\prime}: E_{g} \rightarrow E_{l_{1}}$ and $\Phi_{2}^{\prime}: E_{f} \rightarrow E_{l_{2}}$ be the maps induced by the homotopy-commutative square (4.1). Then the fibres of $\Phi_{1}^{\prime}$ and $\Phi_{2}^{\prime}$ are homotopy-equivalent to those of $\xi$.

\section{§. The dual EHP sequence}

In this section we construct, for a triad $A \stackrel{f}{\longrightarrow} Y \stackrel{g}{-} B$, a dual of the EHP sequence and examine its behaviour. The dual EHP cohomology sequence was first defined by G. W. Whitehead [15] and has been extended by TsuchidaAndo [14].

First, consider the map $\mu: E_{f}^{-} \times E_{g} \rightarrow E_{f, g}$ defined by

$$
\mu((a, \alpha),(\beta, b))=(a, \alpha+\beta, b)
$$

for $a \in A, b \in B,--\alpha, \beta \in E Y$, and the "projections" $\Pi_{1}: E_{f}^{-} \times E_{g} \rightarrow E_{f, g}, \Pi_{2}$ : $E_{f}^{-} \times E_{g} \rightarrow E_{f, g}$ defined by 


$$
\begin{aligned}
& \Pi_{1}((a, \alpha),(\beta, b))=(a, \alpha, *), \\
& \Pi_{2}((a, \alpha),(\beta, b))=(*, \beta, b) .
\end{aligned}
$$

We say that an element $\rho \in \pi\left(S E_{f, g}, V\right)$ is primitive with respect to $\mu$ if and only if $(S \mu) *(\rho)=\left(S \Pi_{1}\right) *(\rho)+\left(S \Pi_{2}\right) *(\rho)$.

Now let

$$
q: E_{f}^{-} * E_{g} \rightarrow S\left(E_{f}^{-} \times E_{g}\right)
$$

be the map which shrinks to a point the ends of the join. We have a map

$$
\mathscr{A}=Q \circ j: E_{f}^{-} * E_{g} \rightarrow S E_{f, g},
$$

where $j: E_{f}^{-} * E_{g} \rightarrow C_{P_{1}, P_{2}}$ and $Q: C_{P_{1}, P_{2}} \rightarrow S E_{f, g}$ are defined in (3.6), (3.2). Then we see at once that $\mathscr{H}=\left(S_{\mu}\right) \circ q$. Note that $\mathscr{H}$ is equivalent to the map obtained from $\mu$ by the Hopf construction. The following lemma allows us to call $\mathscr{H}^{*}$ the dual Hopf invariant associated with the $\operatorname{triad} f, g$.

Lemma 5.1. (cf. [10, Theorem 1]) $\rho \in \pi\left(S E_{f, g}, V\right)$ is primitive with respect to $\mu$ if and only if $\mathscr{A}^{*}(\rho)=0$.

Proof. We consider the diagram associated with the join $E_{f}^{-} * E_{g}$ :

$$
\pi\left(E_{f}^{-} * E_{g}, V\right) \stackrel{q^{*}}{\longleftarrow} \pi\left(S\left(E_{f}^{-} \times E_{g}\right), V\right) \overbrace{\left(S p_{2}\right)^{*}} \pi\left(S E_{g}, V\right) .
$$

Then, by Lemma 1.1', $q^{*} \circ(S \mu)^{*}(\rho)=0$ if and only if there exist $\alpha \in \pi\left(S E_{f}^{-}, V\right)$, $\beta \in \pi\left(S E_{g}, V\right)$ such that

$$
(S \mu)^{*}(\rho)=\left(S p_{1}\right)^{*}(\alpha)+\left(S p_{2}\right)^{*}(\beta) .
$$

Suppose first that the latter equality holds. We denote the injections $E_{f}^{-} \rightarrow E_{f}^{-} \times E_{g}, E_{g} \rightarrow E_{f}^{-} \times E_{g}$ by $i_{1}, i_{2}$ respectively. Applying $\left(S p_{1}\right)^{*}\left(S i_{1}\right)^{*}$ to both sides, we obtain $\left(S \Pi_{1}\right)^{*}(\rho)=\left(S p_{1}\right)^{*}(\alpha)$. Similarly, $\left(S \Pi_{2}\right)^{*}(\rho)=\left(S p_{2}\right)^{*}(\beta)$. This proves that $\rho$ is primitive.

Conversely, since $\Pi_{k}=\left(\Pi_{k} \circ i_{k}\right) \circ p_{k}, k=1,2$, "primitive" implies the existence of $\alpha, \beta$ such that $(S \mu)^{*}(\rho)=\left(S p_{1}\right)^{*}(\alpha)+\left(S p_{2}\right)^{*}(\beta)$. q.e.d.

We now describe an approximation to the fibre and cofibre of $\xi^{\prime}$ by means of the cofibres of $f, g$. Let 


$$
\mu^{\prime}: C_{P_{1}, P_{2}} \rightarrow C_{P_{1}} \vee C_{P_{2}}
$$

be the map obtained by shrinking the "center" $E_{f, g} \times \frac{1}{2}$ of the cylinder part of $C_{P_{1}, P_{2}}$, and let $\Phi_{1}: C_{P_{1}} \rightarrow C_{g}, \emptyset_{2}: C_{P_{2}} \rightarrow C_{f}$ be as in Theorem 3.8. Let

$$
k_{1}: Y \rightarrow C_{f} \text { and } k_{2}: Y \rightarrow C_{g}
$$

denote natural injections and let

$$
\sigma_{1}: E_{f}^{-} \rightarrow \Omega C_{f} \quad \text { and } \sigma_{2}: E_{g} \rightarrow \Omega C_{g}
$$

denote the (Freudenthal) suspension maps given by

$$
\sigma_{1}(a, \alpha)=-\alpha-\hat{a}, \quad \sigma_{2}(\beta, b)=\beta-\hat{b}
$$

for $a \in A, b \in B, \alpha, \beta \in Y^{I}$.

Introduce the diagram

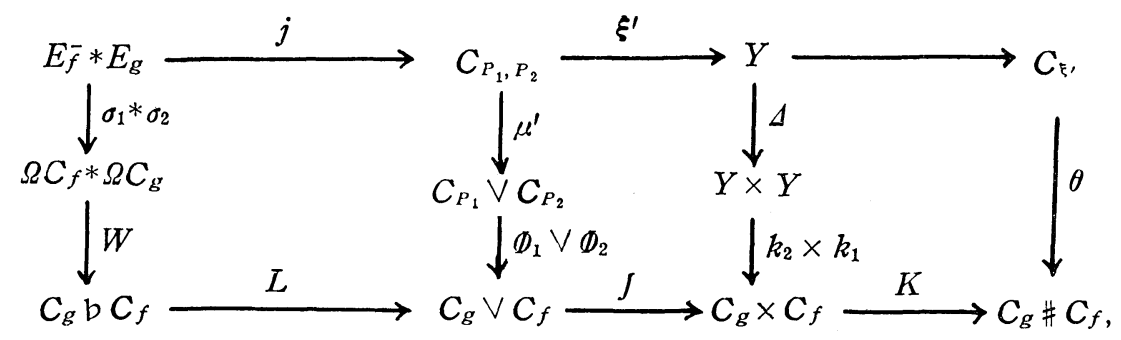

where $W$ is the map defined in (2.7) and $\Delta$ is the diagonal injection. That homotopy-commutativity holds in the middle square, i.e., $\left(k_{2} \times k_{1}\right) \circ \Delta \circ \xi^{\prime} \simeq J$ 。 $\left(\Phi_{1} \vee \Phi_{2}\right) \circ \mu^{\prime}$, can be verified by taking the following homotopy:

$$
\begin{aligned}
& (a, \gamma, b ; t) \rightarrow[(\gamma-\hat{b})+*]\left(\frac{t+3 u t}{4}\right) \times[*+(\hat{a}+\gamma)]\left(\frac{t+3 u t+3-3 u}{4}\right) \\
& a \rightarrow f(a) \times[*+(\hat{a}+\gamma)]\left(\frac{3-3 u}{4}\right), b \rightarrow[(\gamma-\hat{b})+*]\left(\frac{1+3 u}{4}\right) \times g(b)
\end{aligned}
$$

where $0 \leqq u \leqq 1, a \in A, b \in B, \gamma \in Y^{l}, 0 \leqq t \leqq 1$. Therefore the map $\theta$ is induced so that the right square be commutative. Moreover, using (3.6), (5.2) and (2.7), we can verify the following:

$$
\left[\left(\Phi_{1} \vee \Phi_{2}\right) \circ \mu^{\prime} \circ j\right]((1-s)(a, \alpha) \oplus s(\beta, b))= \begin{cases}(\alpha+\beta)(4 s) \in C_{g} & 0 \leqq 4 s \leqq 1 \\ (b, 2-4 s) \in C_{g} & 1 \leqq 4 s \leqq 2 \\ (a, 4 s-2) \in C_{f} & 2 \leqq 4 s \leqq 3 \\ (\alpha+\beta)(4 s-3) \in C_{f} & 3 \leqq 4 s \leqq 4\end{cases}
$$


$\left[L \circ W \circ\left(\sigma_{1} * \sigma_{2}\right)\right]((1-s)(a, \alpha) \oplus s(\beta, b))= \begin{cases}\beta(4 s) \in C_{g} & 0 \leqq 4 s \leqq 1, \\ \hat{b}(2-4 s) \in C_{g} & 1 \leqq 4 s \leqq 2, \\ \hat{a}(4 s-2) \in C_{f} & 2 \leqq 4 s \leqq 3, \\ \alpha(4 s-3) \in C_{f} & 3 \leqq 4 s \leqq 4 .\end{cases}$

It follows that homotopy-commutativity holds in the left square.

The middle square of (5.3) induces the map $\chi: E_{\xi} \rightarrow C_{g} b C_{f}$. We see at once from (5.4) that the composite $E_{f}^{-} * E_{g} \stackrel{F}{\longrightarrow} E_{\xi}, \stackrel{\chi}{\longrightarrow} C_{g} \mathrm{~b} C_{f}$ is given as follows:

$$
(\chi \circ F)((1-s)(a, \alpha) \oplus s(\beta, b))= \begin{cases}\left(-\alpha_{2 s, 1}+\tau\right) \times\left(-\alpha_{2 s, 1}+\rho\right) & 0 \leqq 2 s \leqq 1 \\ \left(\beta_{0,2 s-1}+\tau\right) \times\left(\beta_{0,2 s-1}+\rho\right) & 1 \leqq 2 s \leqq 2\end{cases}
$$

where

$$
\tau=[((\alpha+\beta)-\hat{b})+*] \frac{s}{4}, s, \quad \rho=[((-\beta-\alpha)-\hat{a})+*] \frac{1-s}{4}, 1-s .
$$

Further we have

$$
\left[W \circ\left(\sigma_{1} * \sigma_{2}\right)\right]((1-s)(a, \alpha) \oplus s(\beta, b))= \begin{cases}(\beta-\hat{b})_{0,2 s} \times(-\alpha-\hat{a}) & 0 \leqq 2 s \leqq 1, \\ (\beta-\hat{b}) \times(-\alpha-\hat{a})_{0,2-2} & 1 \leqq 2 s \leqq 2 .\end{cases}
$$

From these results we infer

Lemma 5.5. $W \circ\left(\sigma_{1} * \sigma_{2}\right)$ is homotopic to $\chi \circ F$.

Lemma 5.6. Suppose that $f$ and $g$ are, respectively, $p$ - and $q$-connected and, further, let $Y$ be r-connected. Then $W \circ\left(\sigma_{1} * \sigma_{2}\right)$ is $[p+q+\min (p, q, r+1)+1]$. connected and $\theta$ is $[p+q+\min (p, q, r)+2]$-connected.

Proof. Since the adjoints of $\sigma_{1}, \sigma_{2}$ are respectively $(p+r+1)-$ and $(q+r$ +1 )-connected, it follows from Lemma 1.4 that $\sigma_{1}$ and $\sigma_{2}$ are respectively $\mathrm{min}$ $(2 p, p+r+1)$-connected and $\min (2 q, q+r+1)$-connected. Thus, by Lemma 2.8, $W,\left(\sigma_{1} * \sigma_{2}\right)$ is $[p+q+\min (p, q, r+1)+1]$-connected. To prove the second half, note that, by Lemma $5.5, \chi$ is $[p+q+\min (p, q, r+1)+1]$-connected. Introduce the homotopy commutative diagram

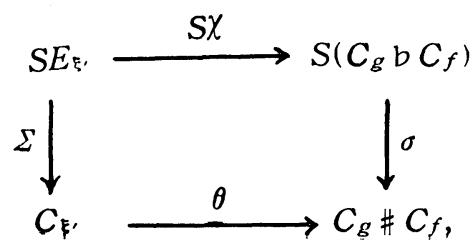


in which the suspension maps $\Sigma, \sigma$ are respectively $(p+q+r+2)-,(p+q+$ $\min (p, q)+3)$-connected. This completes the proof of the second half.

Lemma 5.7. The composition

$$
S\left(E_{f}^{-} * E_{g}\right) \stackrel{S F}{\rightarrow} S E_{\xi}, \stackrel{\Sigma}{\longrightarrow} C_{\xi^{\prime}} \stackrel{\zeta^{\prime}}{\rightarrow} C_{\eta^{\prime}} \stackrel{\partial}{\longrightarrow} S^{9} E_{f, g}
$$

is homotopic to $S_{\subset} \not /: S\left(E_{f}^{-} * E_{g}\right) \rightarrow S^{2} E_{f, g}$, where $\zeta^{\prime}$ is the equivalence in (3.2), $\partial$ the map which results from shrinking $C_{f_{\vee} g}$ and $\Sigma$ the suspension map given by

$$
\begin{aligned}
& \sum(\tau ; a, \gamma, b, s ; t)= \begin{cases}\tau(2-2 t) \in Y & 1 \leqq 2 t \leqq 2, \\
(a, \gamma, b, s ; 2 t) \in C C_{P_{1}, P_{2}} & 0 \leqq 2 t \leqq 1,\end{cases} \\
& \sum(\tau ; a ; t)=(a, 2 t) \text { if } 2 t \leqq 1, \quad=\tau(2-2 t) \text { if } 2 t \geqq 1, \\
& \sum(\tau ; b ; t)=(b, 2 t) \text { if } 2 t \leqq 1, \quad=\tau(2-2 t) \text { if } 2 t \geqq 1
\end{aligned}
$$

for $a \in A, b \in B, r \in Y^{1}, 0 \leqq s \leqq 1,0 \leqq t \leqq 1, \tau \in E Y$.

Proof. In the following diagram, the squares are homotopy-commutative:

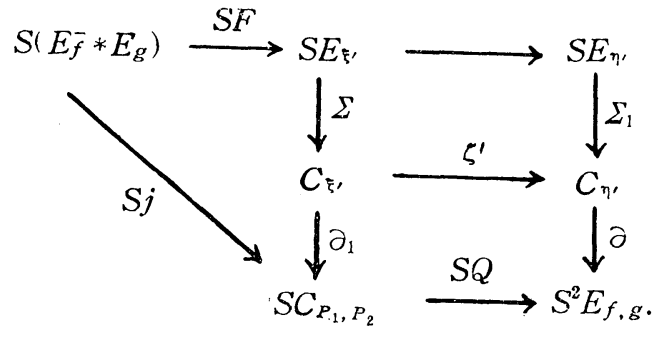

Since $\partial_{1} \circ \Sigma \circ S F$ is given, explicitly, by

$$
((1-s)(a, \alpha) \oplus s(\beta, b), t) \rightarrow \begin{cases}(a, \alpha+\beta, b, s ; 2 t) & 0 \leqq 2 t \leqq 1 \\ * & 1 \leqq 2 t \leqq 2\end{cases}
$$

we see that homotopy-commutativity holds in the left triangle by (3.6). From $\mathscr{A}=Q \circ j$, follows the conclusion of the lemma.

Let $e: C_{f_{\nabla} g} \rightarrow C_{r^{\prime}}$ and $e^{\prime}: Y \rightarrow C_{\xi^{\prime}}$ denote canonical embeddings. Combining Lemmas 5.6, 5.7 with Puppe's sequence associated with $\eta^{\prime}$, we obtain the following reuslt.

THEOREM 5.8. If $f, g$ and $Y$ are respectively, $p$-, $q$ - and $r$-connected, and if $A, B$ and $Y$ have the homotopy type of $C W$-complexes, then for any 1-connected space $V$ such that $\pi_{i}(V)=0$ for $i \geqq p+q+r+2$, the following sequence is exact: 


$$
\begin{aligned}
\pi\left(S E_{f, g},\right. & V) \stackrel{\mathscr{E}^{*}}{\stackrel{(S \mathscr{C}}{*}} \pi\left(C_{f \nabla g}, V\right) \stackrel{\mathscr{Q}^{*}}{\leftarrow} \pi\left(S\left(E_{f}^{-} * E_{g}\right), V\right) \stackrel{(S \mathscr{H})^{*}}{\longleftarrow} \pi\left(S^{2} E_{f, g}, V\right) \\
& \stackrel{(S)^{*}}{\longleftarrow} \pi\left(S C_{f \nabla g}, V\right) \stackrel{(S \mathscr{Q})^{*}}{\longleftarrow} \pi\left(S^{2}\left(E_{f}^{-} * E_{g}\right), V\right) \longleftarrow \cdots,
\end{aligned}
$$

where $\mathscr{E}^{*}$ is $\left(\eta^{\prime}\right)^{*}$ and $\mathscr{Q}^{*}$ denotes $e^{*} \circ\left(\zeta^{\prime} \circ \Sigma \circ S F\right)^{*-1}$. Further, if $\pi_{i}(V)=0$ for $i \geqq p+q+r+3$, then the sequence

$$
\pi\left(E_{f}^{-} * E_{g}, \Omega V\right) \stackrel{\mathscr{A}^{*}}{\longleftarrow} \pi\left(S E_{f, g}, \Omega V\right) \stackrel{\mathscr{E}^{*}}{\longleftarrow} \pi\left(C_{f \nabla g}, \Omega V\right) \stackrel{\mathscr{Q}^{*}}{\longleftarrow} \ldots
$$

is exact.

Note that $\mathscr{Q}^{*}\left(\rho_{1}\right)=\mathscr{Q}^{*}\left(\rho_{2}\right)$ for $\rho_{1}, \rho_{2} \in \pi\left(S\left(E_{f}^{-} * E_{g}\right), V\right)$ if and only if $\rho_{2}=$ $\mathscr{A}^{*}(\tau)+\rho_{1}$ for some $\tau \in \pi\left(S^{2} E_{f, g}, V\right)$.

As an application of Theorem 5.8 we get.

Proposition 5.9. Let $A$ and $B$ be, respectively, $p$ - and $q$-connected. Then the map $\Lambda: \Omega A * \Omega B \rightarrow S(B \hat{*} A)$ defined by

$$
\Lambda((1-t) \alpha \oplus t \beta)=(\alpha+\beta, t),
$$

is $(p+q+\min (p, q))$-connected.

Proof. Consider the triad $B \stackrel{i_{1}}{\longrightarrow} B \vee A \stackrel{i_{2}}{\longleftarrow} A$. It follows from the theorem of Blakers-Massey that the maps $\Phi_{1}^{\prime}: \Omega A \rightarrow E_{i_{1}}^{-}, \Phi_{2}^{\prime}: \Omega B \rightarrow E_{i_{2}}$ are $(p+q-1)$ connected (cf. Theorem 4.6), where $\Phi_{1}^{\prime}, \Phi_{2}^{\prime}$ are both induced by the commutative diagram

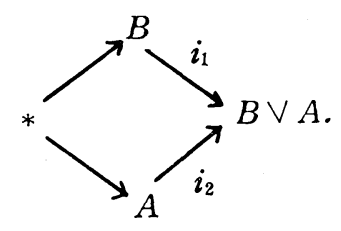

Since $B \vee A$ is $\min (p, q)$-connected and $C_{i_{1} \nabla i_{2}}$ is contractible, it follows from Theorem 5.8 that $\mathscr{A}: E_{i_{1}}^{-} * E_{i_{2}} \rightarrow S E_{i_{1}, i_{2}}=S(B \hat{*} A)$ is $(p+q+\min (p, q)+1)$-connected.

We see that the composite

$$
\Omega A * \Omega B \stackrel{\Phi_{1}^{\prime} * \Phi_{2}^{\prime}}{\longrightarrow} E_{i_{1}}^{-} * E_{i_{2}} \stackrel{\mathscr{A}}{\longrightarrow} S(B \hat{*} A)
$$

is just 1 . This completes the proof, noticing that $\emptyset_{1}^{\prime} * \Phi_{2}^{\prime}$ is $(p+q+\min (p, q))$ connected. 
The above proposition enables us to obtain the following result mentioned at the end of $\S 2$.

Corollary 5.10. $W^{\prime}: A \# B \rightarrow S A \hat{*} S B$, as defined in (2.9), is $(p+q+\min$ $(p, q)+2)$-connected, if $A$ and $B$ are respectively $p$-and $q$-connected.

Proof. Consider the commutative diagram

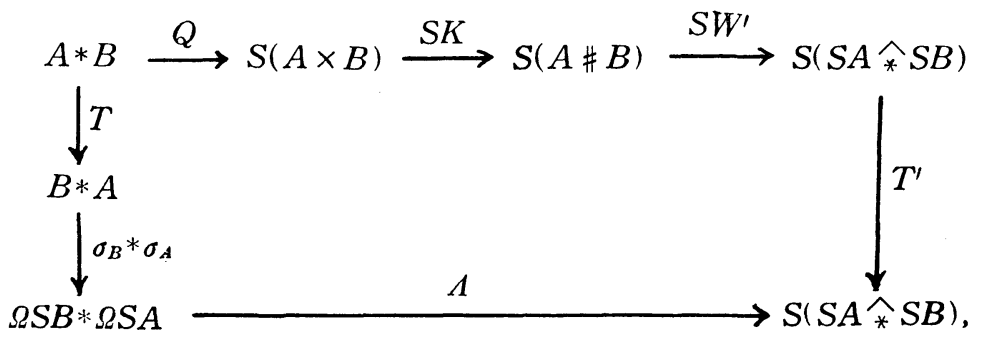

in which $T$ is the switching map, $\Lambda$ the map as defined in Proposition 5.9, $T^{\prime}$ the involution resulting from inversing suspension parameter, and $\sigma_{A}, \sigma_{B}$ are defined by $\sigma_{A}(a)=\hat{a}, \sigma_{B}(b)=-\hat{b}$. Since $\sigma_{B} * \sigma_{A}$ is $(p+q+\min (p, q)+3)$-connected and $S K \circ Q$ is a weak equivalence by Proposition $2.2^{\prime}$, we get the desired conclusion.

Lemma 5.11. Let $\varepsilon: Y \rightarrow \Omega S Y$ denote the canonical embedding, $\varepsilon(y)=\hat{y}$. Let $W^{\prime}: C_{g} \# C_{f} \rightarrow S C_{g} \hat{*} S C_{f}$ be the map described in (2.9). Then the homotopy class of the composition

$$
Y \stackrel{e^{\prime}}{\rightarrow} C_{\xi^{\prime}} \stackrel{\theta}{\longrightarrow} C_{g} \# C_{f} \stackrel{W^{\prime}}{\longrightarrow} S C_{g} \hat{*} S C_{f}
$$

coinsides with the cojoin product $\left\langle\left(\Omega S k_{2}\right) \circ \varepsilon,\left(\Omega S k_{1}\right) \circ \varepsilon\right\rangle$, where $k_{1}: Y \rightarrow C_{f}, k_{2}$ : $Y \rightarrow C_{g}$ are inclusions.

Proof. This follows from

$$
\begin{aligned}
{\left[\left(W^{\prime} \circ \theta \circ e^{\prime}\right)(y)\right](t) } & =\left[\left(W^{\prime} \circ K \circ\left(k_{2} \times k_{1}\right) \circ \Delta\right)(y)\right](t) \\
& = \begin{cases}(y, 1-2 t) \in S C_{f} & 0 \leqq 2 t \leqq 1, \\
(y, 2 t-1) \in S C_{g} & 1 \leqq 2 t \leqq 2 .\end{cases}
\end{aligned}
$$

With the above preliminaries, we can establish the dual EHP sequence for a triad $A \stackrel{f}{\longrightarrow} Y \stackrel{g}{\longleftarrow} B$. 
THEOREM 5.12. Let $A \stackrel{f}{\longrightarrow} Y \stackrel{g}{-} B$ be a triad in which $A, B, Y$ have the homotopy type of $C W$-complexes. If $f, g$ and $Y$ are respectively $p-, q$ - and $r$-connected, then the diagram

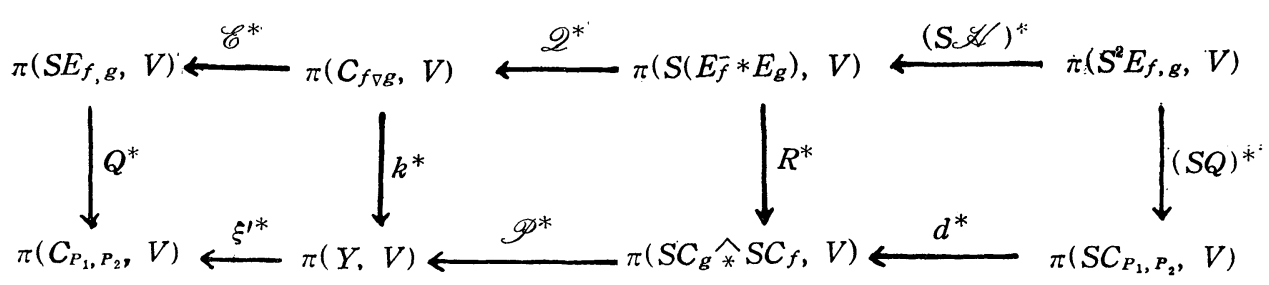

commutes and exact rows for 1-connected space $V$ such that $\pi_{i}(V)=0$ for $i \geqq p$ $+q+\min (p, q, r)+2$, where $\mathscr{P}^{*}$ is the map induced by $\left\langle\left(\Omega S k_{2}\right)_{\circ} \varepsilon,\left(\Omega S k_{1}\right)^{\circ} \varepsilon\right\rangle$ and $R^{*}=\left(W^{\prime} \circ \theta \circ \Sigma \circ S F\right)^{*-1}$ is bijective.

Proof. Note that $W^{\prime}: C_{g} \# C_{f} \rightarrow S C_{g} \hat{*} S C_{f}$ is $(p+q+\min (p, q)+2)$-connected. Then we see that the theorem follows from (3.2), Lemmas $56,5.11$.

Corollary 5.13. If $Y$ is r-connected, then, for a 1-connested space $V$ such that $\pi_{i}(V)=0$ for $i \geqq 3 r+2$, we have an exact sequence:

$$
\begin{aligned}
& \pi(S \Omega Y, V) \stackrel{\mathscr{C}^{*}}{\longleftarrow} \pi(Y, V) \longleftarrow \pi(S(\Omega Y * \Omega Y), V) \stackrel{(S \mathscr{L})^{*}}{\longleftarrow} \pi\left(S^{2} \Omega Y, V\right) \\
& \mathscr{P}^{*}=\langle\varepsilon, \varepsilon\rangle * \cdot \underbrace{R^{*} \downarrow}_{\pi(S Y \hat{*} S Y, V)} \\
& W^{\prime *} \approx \\
& \pi(Y \# Y, V) \text {. }
\end{aligned}
$$

This follows by applying Theorem 5.12 to the triad $* \rightarrow Y \leftarrow *$.

In case where $V$ is the Eilenberg-MacLane space in Corollary $5.13, \mathscr{P}$ can be described in terms of cup-products in the light of Lemma 2.5 and Proposition 2.6.

Finally, we shall furnish $\mathscr{E}^{*}$ with some meaning. Consider the situation (3.1). Let $v: C_{f_{\vee} g} \rightarrow V$ be given and write $u: Y \rightarrow V$ for the composite $Y \stackrel{k}{\longrightarrow} C_{f_{\nabla} g} \stackrel{v}{\longrightarrow} V . \quad v$ gives rise to liftings $\tilde{f}: A \rightarrow E_{u}, \widetilde{g}: B \rightarrow E_{u}$. Let us denote the action of $\Omega V$ on $E_{u}$ by $m: \Omega V \times E_{u} \rightarrow E_{u}$. Then we get.

Proposition 5.14. Let $\tau$ denote the adjoint of $\eta^{\prime *}(v)$. Then

$$
m_{*}\left\{\tau, P_{2}^{*}(\widetilde{g})\right\}=P_{1}^{*}(\tilde{f}) .
$$


Moreover, given $h: K \rightarrow A, k: K \rightarrow B$ with $f \circ h \simeq g \circ k$, we can find $l: K \rightarrow$ $E_{f, g}$ such that $P_{1} \circ l \simeq h, P_{2} \circ l \simeq k$. We see easily that the composite

$$
S K \stackrel{S l}{\longrightarrow} S E_{f, g} \stackrel{\eta^{\prime}}{\longrightarrow} C_{f_{\nabla} g} \longrightarrow S A \vee S B,
$$

where the last arrow is the identification map resulting by shrinking $Y$ to a point, is homotopic to the difference $j_{1} \circ(S h)-j_{2} \circ(S k)$, where $j_{1}: S A \rightarrow S A \vee S B$, $j_{2}: S B \rightarrow S A \vee S B$ are inclusions. Thus, in case $K$ is a suspension, $v^{\circ} \eta^{\prime} \circ(S l)$ represents the generalized Toda bracket $\left\{\begin{array}{l}f h \\ g-k\end{array}\right\}$ (see [5]).

Further, we assume $f \circ h \simeq g \circ k \simeq 0$. Then $h, k$ can be lifted to $\widetilde{h}: K \rightarrow E_{f}^{-}$, $\widetilde{k}: K \rightarrow E_{g}$. We may choose the composite

$$
K \stackrel{\{\widetilde{h}, \widetilde{k}\}}{\longrightarrow} E_{f}^{-} \times E_{g} \stackrel{\mu}{\longrightarrow} E_{f, g}
$$

for $l$. As $\eta^{\prime} \circ \mathscr{A} \simeq 0, v \circ \eta^{\prime}$ is primitive with respect to $\mu$. Therefore we get

$$
v \circ \eta^{\prime} \circ(S l) \simeq v \circ \eta^{\prime} \circ\left(S \Pi_{1}\right) \circ S\{\widetilde{h}, \widetilde{k}\}+v \circ \gamma^{\prime} \circ\left(S \Pi_{2}\right) \circ S\{\widetilde{h}, \widetilde{k}\} .
$$

A simple caiculation shows

PROPOSITION 5.15. $\quad v \circ \eta^{\prime} \circ(S l): S K \rightarrow V$ represents the difference $-u_{f}(h)+u_{g}$ ( $k$ ) of functional u-operations.

\section{§ 6. The EHP sequence}

This section studies the situation dual to that considered in $\S 5$. Namely, by generalizing a result of Ganea [8] to a cotriad, we will regain "symmetry".

Let $A \stackrel{f}{\longleftarrow} X \stackrel{g}{\rightarrow} B$ be a cotriad and consider the associated diagram (4.1). The notations of $\$ 4$ will be used without specific mention.

First, we try to seek an approximation to the fibre and to the cofibre of $\xi$. Introduce the diagram

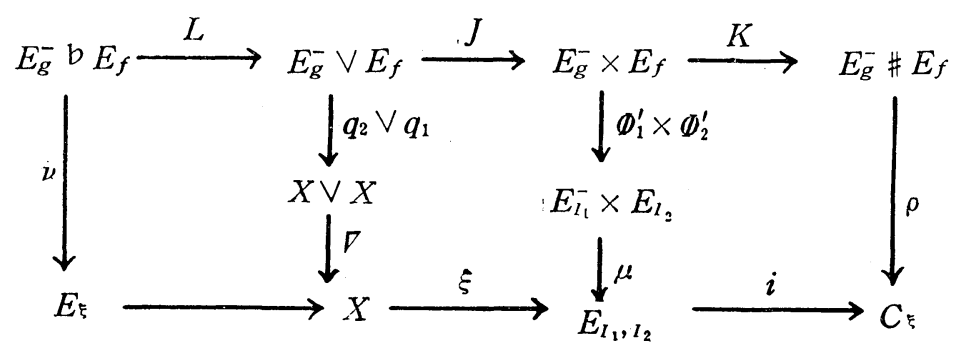


in which $\mu$ is the "multiplication" defined at the beginning of $\S 5, \nabla$ the folding map, $\Phi_{1}^{\prime}$ and $\Phi_{2}^{\prime}$ the maps as defined in Theorem 4.6, and $q_{2}, q_{1}$ are the projections. It -is easily seen that the middle square homotopy-commutes, and hence induces the maps $\rho, \nu$.

THEOREM 6.2. Let $f, g$ be respectively $p$-, q-connected and let $X$ be $r$-connected. Then $\rho$ is $[p+q+\min (p, q, r+1)-1]$-connected and $\nu$ is $[p+q+\min (p, q, r$ $+1)-2]$-connected.

Proof. Apply the suspension functor to the right square and then augment as follows:

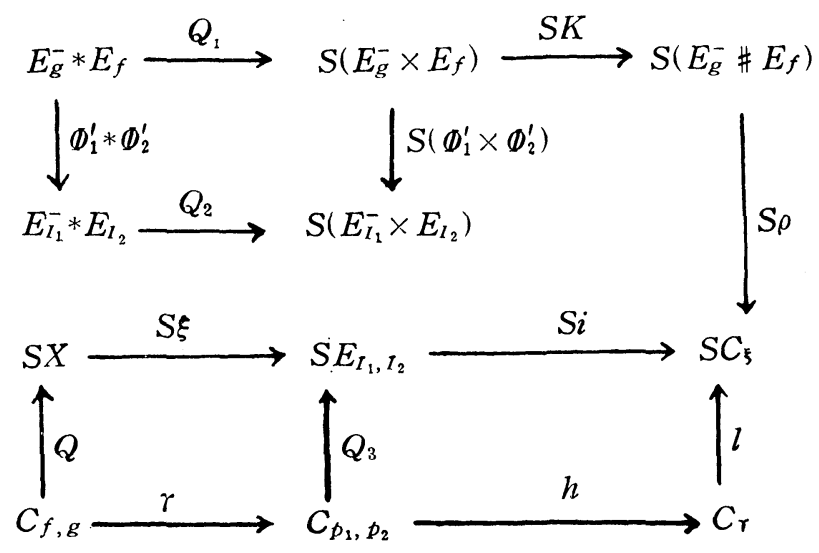

in which $p_{1}: E_{1_{1}, I_{2}} \rightarrow A, p_{2}: E_{1_{1}, l_{2}} \rightarrow B$ are projections, $\gamma$ the map determined by the commutative diagram:

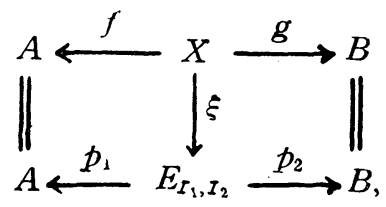

and $l$ the map induced by the identification maps $Q, Q_{3}$. It follows from $1.3^{\prime}$ that $l$ is a weak homotopy equivalence, since $C_{r}$ is homotopy-equivalent to the mapping cylinder of a cotriad $* \leftarrow C_{\xi} \rightarrow *$. Also, by Theorems 4.3 and 4.6, $\Phi_{1}^{\prime} * \Phi_{2}^{\prime}$ is $(p+q+\min (p, q))$-connected.

Define a map $\xi^{\prime}: C_{p_{1}, t_{2}} \rightarrow C_{f, g}$ as the canonical extension of a triad $A \stackrel{I_{1}}{\longrightarrow} C_{f, g} \stackrel{I_{2}}{-} B$ (see $\S 3$ ). We see that $\xi^{\prime} \circ \gamma=$ identity. Since the fibre of $\xi^{\prime}$ is 
equivalent to $E_{1_{1}}^{-} * E_{1_{2}}$, by Theorem 3.4 , we get the following commutative diagram

$$
\begin{aligned}
& 0 \rightarrow H_{k}\left(E_{I_{1}}^{-} * E_{I_{2}}\right) \stackrel{j_{*}}{\longrightarrow} H_{k}\left(C_{p_{1}, p_{2}}\right) \stackrel{\xi_{*}^{\prime}}{\longrightarrow} H_{k}\left(C_{f, g}\right) \rightarrow 0 \\
& 0 \leftarrow H_{k}\left(C_{\curlyvee}\right) \stackrel{h_{*}}{\longleftarrow}-H_{k}\left(C_{p_{1}, p_{2}}\right) \stackrel{\gamma_{*}}{\longleftarrow} H_{k}\left(G_{f, g}\right) \leftarrow 0,
\end{aligned}
$$

in which the rows are exact for $k \leqq p+q+\min (p, q, r+1)+1$. Chasing this diagram, we conclude thet $h \circ j$ is $(p+q+\min (p, q, r+1)+1)$-connected.

Now, since $Q_{3} \circ j=S \mu^{\circ} Q_{2}$ by (3.6), homotopy-commutativity of (6.3) implies

$$
\begin{aligned}
S \rho \circ S K \circ Q_{1} & \simeq S i \circ S_{\mu} \circ Q_{2} \circ\left(\emptyset_{1}^{\prime} * \Phi_{2}\right) \\
& =S i \circ Q_{3} \circ j \circ\left(\emptyset_{1}^{\prime} * \emptyset_{2}^{\prime}\right) \simeq l \circ h \circ j \circ\left(\emptyset_{1}^{\prime} * \Phi_{2}^{\prime}\right) .
\end{aligned}
$$

Upon noticing that $S K \circ Q_{1}$ is a weak equivalence by Proposition 2.2', we infer that $S \rho$ is $[p+q+\min (p, q, r+1)]$-connected.

Finally, the connectivity of $\nu$ follows from the homotopy-commutative diagram

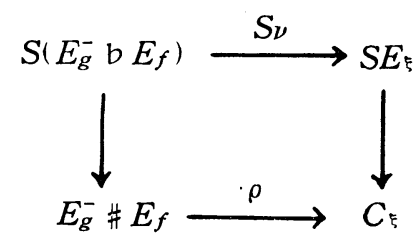

where the vertical maps are "suspension maps", the left of which is $[p+q+$ $\min (p, q)]$-connected, whereas, the right is $[p+q+\min (r, p+q-1)]$-connected.

q.e.d.

Next, using the map $\mu^{\prime}: C_{f, g} \rightarrow C_{f} \vee C_{g}$ which results from shrinking the center of cylinder to a point, we define the Hopf invariant

$$
H: \Omega C_{f, g} \rightarrow C_{f} \hat{*} C_{g}
$$

associated with a cotriad $f, g$ as the composition

$$
\Omega C_{f, g} \stackrel{\Omega_{\mu^{\prime}}}{\longrightarrow} \Omega\left(C_{f} \vee C_{g}\right) \stackrel{I}{\longrightarrow} C_{f} \hat{*} C_{g} .
$$

The following is dual to Lemma 5.1.

Lemma 6.4. Let $r_{1}: C_{f, g} \rightarrow C_{f} \vee C_{g}, r_{2}: C_{f, g} \rightarrow C_{f} \vee C_{g}$ be the "injections" which are respectively the compositions of $C_{f, g} \rightarrow C_{f}, C_{g}$ (projections) with $C_{f}$, 
$C_{g} \rightarrow C_{f} \vee C_{g}$. Then $H_{*}(\tau)=0$ for $\tau \in \pi\left(V, \Omega C_{f, g}\right)$ if and only if the equality $\left(\Omega_{\mu^{\prime}}\right)_{*}(\tau)=\left(\Omega r_{1}\right)_{*}(\tau)+\left(\Omega r_{2}\right)_{*}(\tau)$ holds.

Now we shall define $F^{\prime}: C_{\xi} \rightarrow C_{f} \hat{*} C_{g}$, dual to the map $F$ defined in (3.5). Put

$$
\begin{array}{lr}
F^{\prime}(x, s)=\left(\mu^{\prime} x\right) \frac{1-s}{2}, \frac{1+s}{2}=-\hat{x}_{0, s}+\hat{x}_{0, s} \quad x \in X, 0 \leqq s \leqq 1, \\
F^{\prime}(\beta)=\mu^{\prime} \beta \quad \beta \in E_{l_{1}, l_{2}} \subset\left(C_{f, g}\right)^{I},
\end{array}
$$

where $-\hat{x}_{0, s} \in\left(C_{f}\right)^{I}, \hat{x}_{0, s} \in\left(C_{g}\right)^{l}$. This corresponds to the map $\mathscr{T}$ defined by Ganea [8]. We see easily that the following diagram is commutative :

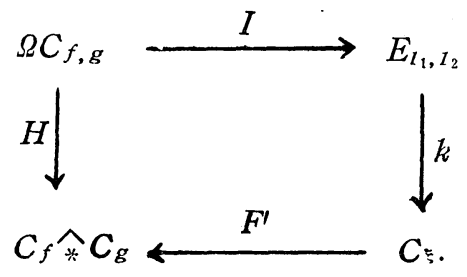

Observe that it seems difficult to define a dual of $G^{\prime}$ given in Theorem 3.4.

Lemma 6.5. The composite map

$$
\Omega^{2} C_{f, g} \stackrel{\text { injection }}{\longrightarrow} E_{\eta} \stackrel{\zeta}{\longrightarrow} E_{\xi} \stackrel{\bar{\sigma}}{\longrightarrow} \Omega C_{\xi} \stackrel{\Omega F^{\prime}}{\longrightarrow} \Omega\left(C_{f} \hat{*} C_{g}\right)
$$

is homotopic to $\Omega H$, where $\bar{\sigma}$ is the suspension map.

Lемма 6.6. The diagram homotopy-commutes:

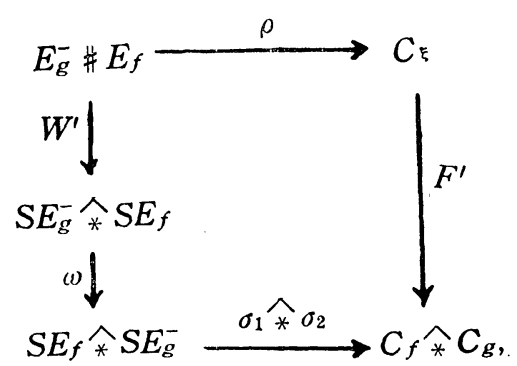

where $\omega$ is the involution switching factors and $\sigma_{1}, \sigma_{2}$ are given as follows:

$$
\begin{aligned}
& \sigma_{1}(\alpha, x ; s)= \begin{cases}\alpha(2 s) & 2 s \leqq 1 \\
(x, 2-2 s) & 2 s \geqq 1\end{cases} \\
& \sigma_{2}(x, \beta ; s)= \begin{cases}\beta(1-2 s) & 2 s \leqq 1 \\
(x, 2-2 s) & 2 s \geqq 1\end{cases}
\end{aligned}
$$


Hence, if $f, g$ and $X$ are respectively $p-, q$ - and $r$-connected, then $F^{\prime}$ is $[p+q+$ $\min (r+1, p, q)-1]$-connected.

Proof. This follows by combining the following facts :

$\rho$ is $[p+q+\min (p, q, r+1)-1]$-connected by Theorem 6.2 ,

$W^{\prime}$ is $[p+q+\min (p, q)-1]$-connected by Corollary 5.10,

$\sigma_{1}, \sigma_{2}$ are respectively $[p+\min (p, r)+1]-,[q+\min (q, r)+1] \cdot$ connected by

Lemma 1.5 .

Lemma 6.7. Let $l_{1}: S \Omega E_{f} \rightarrow X, l_{2}: S \Omega E_{g}^{-} \rightarrow X$ be respectively the composite maps of canonical ones:

$$
S \Omega E_{f} \longrightarrow E_{f} \stackrel{q_{1}}{\longrightarrow} X, \quad S \& E_{g}^{-} \longrightarrow E_{g}^{-} \stackrel{q_{2}}{\longrightarrow} X .
$$

Then the homotopy class of the composition

$$
\Omega E_{f} * \Omega E_{g}^{-} \stackrel{t * t}{\longrightarrow} \Omega E_{f} * \Omega E_{g}^{-} \stackrel{W}{\longrightarrow} E_{g}^{-} \mathrm{b} E_{f} \stackrel{\nu}{\longrightarrow} E_{\xi} \stackrel{\text { projection }}{\longrightarrow} X
$$

coincides with the generalized Whitehead product $\left[l_{1}, l_{2}\right]$, where $t$ denote inversions.

This follows from the fact that the above composition is equal to $\nabla \circ\left(q_{2}\right.$ $\left.\vee q_{1}\right) \circ L \circ W \circ(t * t)$.

Combining Lemmas $6.5,6.6,6.7$ with Theorem 6.2 and noting that $\vec{\sigma}$ is $(p+q+r-1)$-connected, we get

THEOREM 6.8. Let $f, g$ and $X$ be $p-, q$ - and $r$-connected respectively, and let $k$ be a positive integer. Then, for any $C W$-complex $K$ with $\operatorname{dim} K+k \leqq p+q+$ $\min (r+1, p, q)-3$, we have the following exact sequence

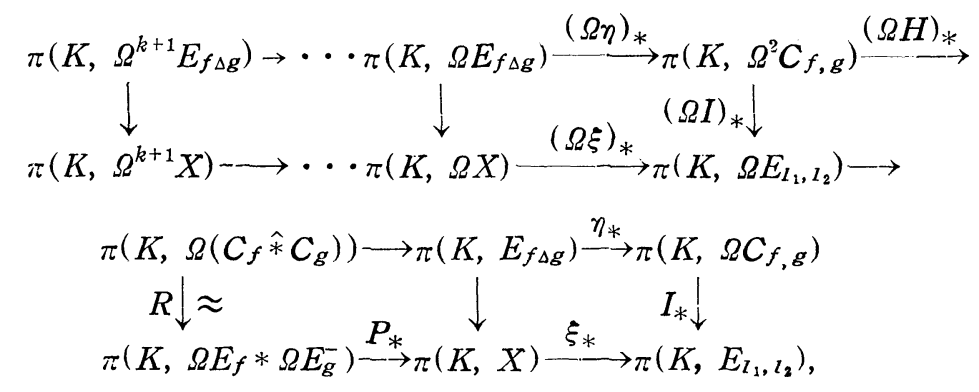

in which $P_{*}$ is the map induced by $\left[l_{1}, l_{2}\right]$ and $R$ the bijection $(t * t)_{*^{\circ}}\left(\Omega F^{\prime} \circ \bar{\sigma} \circ\right.$ $\nu \circ W)_{*}{ }^{-1}$. 


\section{REFERENCES}

[1] S. Araki, On the triad excision theorem of Blakers and Massey, Nagoya Math. J. 6 (1953), 129-136.

[2] M. Arkowitz. The generalized Whitehead product, Pacific J. Math. 12 (1962), 7-23.

[ 3 ] M. Arkowitz, Commutators and cup products, Illinois J. Math. 8 (1964), 571-581.

[4] W. D. Barcus and J.P. Meyer, The suspension of a loop space, Amer. J. Math. 80 (1958), 895-920.

[ 5 ] M. G. Barratt, Homotopy operations and homotopy groups, A. M. S. Summer Topology Institute, Seattle (1963).

[6] I. Berstein and P. J. Hilton, On suspensions and comultiplications, Topology 2 (1963), 73-82.

[7] T. Ganea, P. J. Hilton and F. P. Peterson, On the homotopy-commutativity of loop-spaces and suspensions, Topology 1 (1962), 133-141.

[8] T. Ganea, A generalization of the homology and homotopy suspension, Comm. Math. Helv. 39 (1965).

[9] P. J. Hilton, Homotopy theory and duality, mimeographed notes, Cornell University (1959).

[10] P. J. Hilton, Note on a theorem of Stasheff, Bull. Acad. Pol. Sci. 10 (1962), 127-130.

[11] J. C. Moore, Some applications of homology theory to homotopy problems, Ann. of Math. 58 (1953), 325-350.

[12] I. Namioka, Maps of pairs in homotopy theory, Proc. London Math. Soc. (3) 12 (1962), 725-738.

[13] Y. Nomura, An application of the path-space technique to the theory of triads, Nagoya Math. J. 22 (1963), 169-188.

[14] K. Tsuchida and H. Ando, On the generalized cohomology suspension, Sci. Rep. Fac. Lit. Sci. Hirosaki Univ. 10 (1963), 35-45.

[15] G. W. Whitehead, On the homology suspension, Ann. of Math. 62 (1955), 254-268.

\section{Nagoya Institute of Technology}

Nagoya, Japan 